\title{
A simple and rapid method to determine the zygosity of uPA-transgenic SCID mice
}

\author{
P. Meuleman, P. Vanlandschoot, and G. Leroux-Roels* \\ Center for Vaccinology, Ghent University, De Pintelaan 185, Ghent 9000, Belgium
}

Received 7 July 2003

\begin{abstract}
Successful transplantation of xenogeneic hepatocytes into uPA-transgenic SCID mice depends on the zygosity of the recipient mice. Normally, the difference between homozygous and heterozygous animals is determined via a quantitative Southern blot. We sequenced a part of the mouse genome that is eliminated upon integration of the transgene in the genome. Based on that sequence we developed a multiplex PCR that allows the unambiguous discrimination of negative, heterozygous, and homozygous uPAtransgenic SCID mice in a single day procedure. The speed of the procedure is an essential quality because transplantation of xenogeneic hepatocytes into uPA-SCID mice should be done as soon as possible after birth.
\end{abstract}

(C) 2003 Elsevier Inc. All rights reserved.

Keywords: uPA; SCID; Transplantation; Hepatocytes; Genotyping; PCR; Animal model; HBV; HCV

Infections with the hepatitis B virus (HBV) and the hepatitis $\mathrm{C}$ virus (HCV) are major health problems. Worldwide chronic infections with HBV and HCV affect an estimated 350 and 170 million people, respectively, and are causing over a million deaths annually [1]. In the past two decades major progress has been made in the prevention and treatment of these infections but the search for more efficacious, tolerable, and affordable treatments for both ailments as well as the quest for a vaccine for $\mathrm{HCV}$ go on. This research is hampered by the lack of simple in vitro culture systems and reliable small animal models. Both HBV and HCV have a very narrow tropism and only man and chimpanzee are natural hosts of these viruses. Ethical and financial constraints limit the use of chimpanzees for biomedical research in general and hepatitis-related research in particular. Therefore a small animal model to study HBV and HCV is badly needed. Mice or rats are the prime candidates to create such a model but the stringent tropism of HBV and HCV imposes that hepatocytes from man or chimpanzee are transferred in these rodents. To allow the survival and expansion of xeno-

\footnotetext{
${ }^{*}$ Corresponding author. Fax : +32-9-240-63-11.

E-mail address: geert.lerouxroels@Ugent.be (G. Leroux-Roels).
}

geneic donor hepatocytes in mouse or rat, the recipient animal must provide an environment that is permissive for the engraftment and the expansion of the liver cells. Both criteria are met in an immune deficient animal that suffers from a severe liver disease. An animal with both ailments is not available but can be created by crossing the Alb-uPA transgenic mouse with a severely immune deficient mouse strain such as the nude $(n u / n u)$, the SCID/beige, or the $\mathrm{RAG}^{-/-}$mouse [2-4].

The Alb-uPA transgenic mouse, developed in 1990 by Heckel et al. [5] to study plasminogen hyperactivation and therapeutic protocols to prevent bleeding, contains a tandem repeat of four murine uPA genes under the control of an albumin promoter. The transgene overexpression results in profound hypofibrinogenemia and accelerated hepatocyte death. Homozygous animals can be rescued by transplantation of normal hepatocytes from mouse [6,7], rat [2], woodchuck [3] or man [4,8] which undergo rapid proliferation to replace the dying hepatocytes. Transplants of hepatocytes from species susceptible to hepatitis viruses can be infected with the homologous hepatitis virus. This was first accomplished with woodchuck hepatocytes infected with the woodchuck hepatitis virus [3] and more recently with human hepatocytes which could be infected with HVB [8] and 
HCV [4]. Animals heterozygous for the uPA transgene spontaneously lose the transgene at low frequency and the ensuing 'rescued' mouse hepatocytes then repopulate the liver [9]. For this reason heterozygous animals are inferior recipients of xenogeneic hepatocyte grafts compared to uPA homozygous mice. A rapid and reliable screening method to discriminate homozygous from heterozygous transgenic mice is therefore important for successful hepatocyte transplantation.

Initially this analysis of zygosity was performed by quantitative comparison of the Southern blot signal produced by probes for an endogenous gene and the transgenic uPA gene [2]. This method is time consuming and may generate ambiguous results. Rhim et al. also noted that the insertion of the uPA transgene into the mouse genome resulted in the deletion of endogenous DNA at the integration site. These investigators pickedup part of this sequence in plasmid p3371C and used this as a probe to analyze the genotype of the animals. Using a multiplex PCR based on this deleted endogenous sequence we designed a fast and reliable assay that allows for the unambiguous determination of the zygosity of the transgenic animals.

\section{Materials and methods}

Breeding of the $u P A-S C I D$ mice. B6SJL-TgN(Alb1Plau)144Bri mice were back-crossed on CBySmn.CB17-Prkdcsid mice. Both strains were purchased from the Jackson Laboratories (Bar Harbor, Maine, USA). Screening for the SCID background was done with an in-house mouse IgM sandwich Elisa. Screening for the uPA transgene was done by PCR, initially using the genotyping protocol described by the Jackson Laboratories [10] and subsequently by the in-house method described herein. All mice were bred and handled under specific pathogen free (SPF) conditions in the SCID mouse facility of the Department of Clinical Chemistry, Microbiology and Immunology, Ghent University.

Amplification, isolation, and sequencing of plasmid p3371C. 'Top 10' Escherichia coli cells (Invitrogen, Merelbeke, Belgium) were transformed with plasmid p3371C (kindly provided by Dr. R. Palmiter) by heat shock and grown overnight on LB-plates containing Ampicillin. Resulting colonies were resuspended in LB-broth containing ampicillin and incubated overnight at $37^{\circ} \mathrm{C}$ on a shaking platform. Cells were centrifuged and plasmid was isolated with a HiSpeed Plasmid purification kit (Qiagen, Hilden, Germany) according to the manufacturer's protocol.

Plasmid p3371C was then sequenced with an ABI 310 sequencer (Applied Biosystems, Lennik, Belgium). Briefly, $500 \mathrm{ng}$ plasmid, 3.2 pmol primer, and $8 \mu \mathrm{l}$ of the Terminator Ready Reaction Mix (Applied Biosystems, Lennik, Belgium) were denatured at $90^{\circ} \mathrm{C}$ for $30 \mathrm{~s}$. Annealing $\left(15 \mathrm{~s}\right.$ at $\left.50^{\circ} \mathrm{C}\right)$ and polymerization $\left(4 \mathrm{~min}\right.$ at $\left.60^{\circ} \mathrm{C}\right)$ were repeated for 25 cycles. The resulting product was then purified using ethanol/sodium acetate precipitation and analyzed on the sequencer.

Isolation of mouse genomic DNA. Genomic DNA was extracted from the mouse tail using the Nucleospin Tissue Kit (BD Biosciences Clontech, Erembodegem, Belgium) according to the manufacturer's protocol. Briefly, tissue from the mouse tail was digested with Proteinase $\mathrm{K}$ at $56^{\circ} \mathrm{C}$ for $3 \mathrm{~h}$ or until the sample was completely lysed. After addition of a chaotropic agent the samples were incubated for $10 \mathrm{~min}$ at $70^{\circ} \mathrm{C}$. Then ethanol was added and the sample was loaded on the Nucleospin columns and centrifuged for $1 \mathrm{~min}$ at $11,000 \mathrm{~g}$. The column containing the trapped DNA was washed twice to remove any contaminating proteins and other cellular debris. After contaminants were spun through the filter, nucleic acids were eluted in $100 \mu \mathrm{l}$ of a Tris-buffered solution. All enzymes and buffers were provided by the manufacturer.

PCR. The PCR mix consists of $49 \mu \mathrm{l}$ of thermophilic DNA polymerase buffer supplemented with $200 \mu \mathrm{M}$ dNTPs (Roche Diagnostics, Vilvoorde, Belgium), $2.5 \mathrm{mM} \mathrm{MgCl} 2,1 \mu \mathrm{M}$ of each primer, and $2.5 \mathrm{U}$ Taq DNA polymerase (Promega, Leiden, The Netherlands). To this mixture $1 \mu \mathrm{l}$ of mouse genomic DNA was added. The PCR cycling profile was as follows: an initial denaturation step of $5 \mathrm{~min}$ at $94^{\circ} \mathrm{C}$ was followed by 30 cycles of $30 \mathrm{~s}$ denaturation at $94^{\circ} \mathrm{C}, 30 \mathrm{~s}$ primer annealing at $62^{\circ} \mathrm{C}$, and $30 \mathrm{~s}$ primer extension at $72^{\circ} \mathrm{C}$. To promote completion of partial extension, the samples were held at $72{ }^{\circ} \mathrm{C}$ for $5 \mathrm{~min}$. The samples were then stored on ice until analysis on a $2.5 \%$ agarose gel.

\section{Results}

\section{Sequencing of plasmid p3371C}

Rhim et al. [2] noted that the insertion of the uPA transgene into the mouse genome resulted in the deletion of endogenous DNA at the integration site. These investigators picked up part of this sequence in plasmid p3371C (Fig. 1) and used the BamHI-fragment of this region as a probe for detection via Southern blotting.

The sequence of the deleted region was unknown but part of the plasmid has been sequenced. Starting from this known sequence we performed serial overlapping sequence reactions until we completely elucidated the code of the deleted region. All the known restriction sites inside p3371C were used as positive markers during sequencing. The Bam HI fragment (underlined in Fig. 1), previously reported to be approximately $550 \mathrm{bp}$ long, consisted of the following $664 \mathrm{bp}$ long sequence:

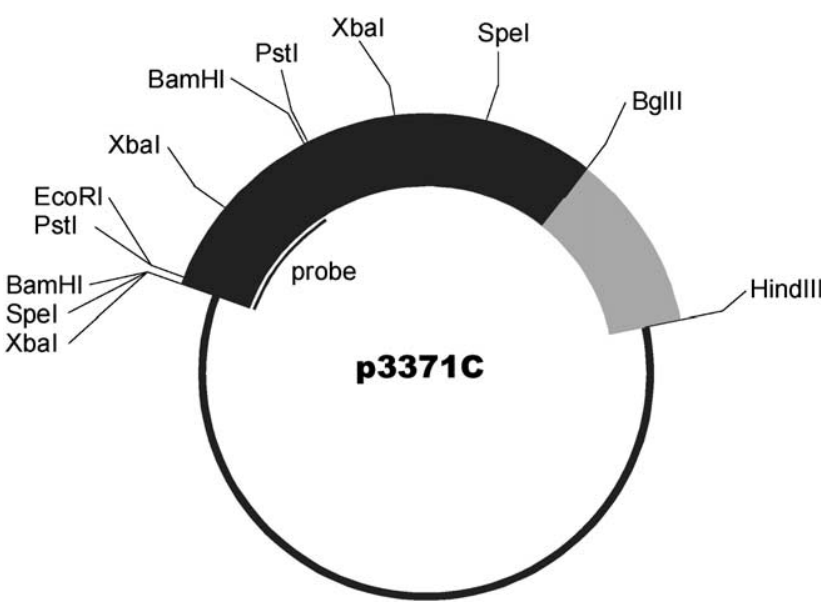

Fig. 1. Map of plasmid p3371C containing a $2 \mathrm{~kb}$ part of the mouse genome which has been deleted at the uPA transgene integration site. The grey bar represents a known sequence which was used as the starting point for the sequencing reactions. All unknown parts are represented by a black bar. Enzymatic digestion of plasmid p3371C with $B a m H I$ produces a $664 \mathrm{bp}$ fragment which was previously used as a probe for Southern blotting. 
GGATCCAGCTCAAGGGAAGGCTCCAAGGCCT GACACTTACTGATGCTATGGTGAATATTCTCTC ATTATAAGGGTGACAGAAATAAGAGAAGAAT TTACATAAAAAGTAAAAGGAACAATGAGTTT AAAGCAGGGGAGTGTATCCACAGTTGAAATA ACTACATTTGTCCTTTAAAACGTGGAATTAATC TTTCTCTTCTCTTGCCCTCTCACATCATTGAATT TTGCATATTAAGATATTTGCATGTCCAAGAAG CAGCTGTTTTTGGAAACTGTTATACTTTCTACT TTTCTCCATTGCTACTCCAATTGCAAAGCACAT CATCACACAATGTGTGAATGATCCAGAGAGTC TGATAGCATATCTCCCAAAGGCAGTCATTTCA AAACTCTAGACAATCACCATCTGCATTGCATG GGCCAGATTTCAAACAGGAGGCTCAAAGTAG ACTCTGGAAATTTGCTGCTGTTGGCTGTCTTG AGGGTCTCAATTGTAGATTCATTTCTCCAAACT ATTGGAAGAATCTATTAATAGGAAAATTAACT TAAAAACACAATTGATAATACCTCTCTTTTTTT TAAAGTGAATTGGACCTTGTGTATTGCACAGA CAGTAAGTTATGAATTTACCAAGACTTAGAAT TCCTGCAGCCCGGGGGATCC.

\section{Multiplex PCR}

Based on the sequence of the deleted region and the nature of the transgene cassette we developed a multiplex PCR based on three primer pairs.

Primer pair one, ApoA\#1: 5'-CATCTCGCACCTTT AGCCAT- $3^{\prime}$ and ApoA\#2: 5'-TCTCTGTGCCCAG GAAGGTA- $3^{\prime}$, is a control primer pair amplifying a $226 \mathrm{bp}$ region of the mouse Apolipoprotein A1-gene. This is a control primer pair that should always result in a positive signal.

Primer pair two, uPA\#1: 5'-CATCCCTGTGACCC CTCC- $3^{\prime}$ and uPA\#2: 5'-CTCCAAAACCACCCCCC TC- $3^{\prime}$, amplifies a 151 bp region within the $3^{\prime}$-untranslated region of the human growth hormone which is present in the transgene cassette. This PCR product allows us to discriminate between uPA negative and positive animals.

The amplification product of primer pair three, uPA\#3: 5'-TTCTCTTCTCTTGCCCTCTCACA- $3^{\prime}$ and uPA\#4: 5'-TTGAGACCCTCAAGACAGCCA-3' (both underlined in the above shown sequence), is $301 \mathrm{bp}$ large and is the product of a region within the mouse genome which was deleted when the transgene had entered.

The combination of the three above-mentioned primer pairs allowed us to determine unambiguously the zygosity of the transgenic animals. Fig. 2 clearly shows that in case of a uPA negative animal (lane 2) two bands appear (226 and $301 \mathrm{bp}$ ). Because the transgene is absent the $151 \mathrm{bp}$ band is not present. Lane 4 shows the PCR products of a homozygous uPA animal. Here also only two bands are visible, the 226 control band and the $151 \mathrm{bp}$ transgene band. There is no amplification product from primer set uPA\#3 and \#4 because the trans-

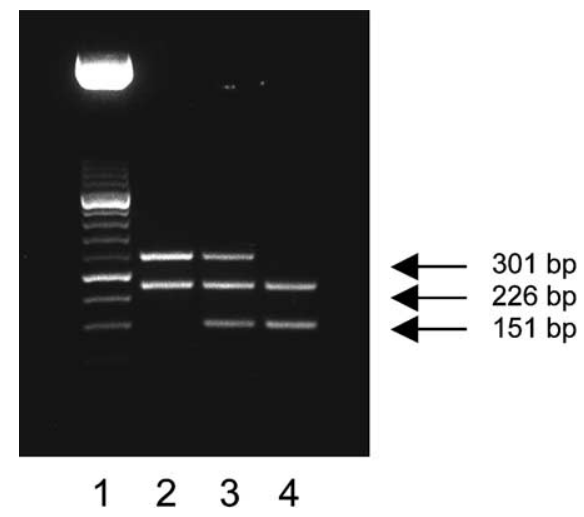

Fig. 2. Multiplex PCR to determine the zygosity of uPA-transgenic mice. Mouse genomic DNA isolated from an $\mathrm{uPA}^{-/-}$(lane 2), $\mathrm{uPA}^{+/-}$ (lane 3), and $\mathrm{uPA}^{+/+}$(lane 4) SCID mouse was used as a template. The $301 \mathrm{bp}$ product is only present in negative and heterozygous animals. The amplification product from the transgene itself (151 bp) is only absent in $\mathrm{uPA}^{-/-}$animals. A control amplification product (226 bp) appears in all samples. All samples were compared to DNA molecular weight marker XIII (Roche, Vilvoorde, Belgium) run in lane 1.

gene has been inserted in both copies of the chromosome. In case of a uPA heterozygous animal (lane 3) all three bands appear. Heterozygous animals still have one chromosome left where the endogenous DNA has not been deleted, explaining the presence of a $301 \mathrm{bp}$ product. This clearly demonstrates the simplicity and the clarity of this method.

\section{Discussion}

The need for an affordable, reliable, and ethically acceptable in vivo model to study HBV and HCV infections is high. The uPA-mouse model first described in 1990 represents one of the most promising tools to create this research tool. uPA-transgenic mice bred on an immune incompetent background like SCID, SCID/ beige or $\mathrm{RAG}^{-1-}$ allow a repopulation of the sick liver by allogeneic and xenogeneic hepatocytes. When transplanted with human hepatocytes these animals become susceptible to infections with $\mathrm{HBV}$ and $\mathrm{HCV}[4,8]$. However, the outcome of the xenogeneic liver cell graft in these animals is largely dependent on the zygosity of the uPA transgene in these animals. Whereas the survival and expansion of the liver cell graft is poor in heterozygous mice, an excellent and sustained engraftment is observed in homozygous animals. Since the success of the transplantation increases when the donor hepatocytes are introduced early after birth, preferably at the age of one to two weeks, a fast and unambiguous method to determine the zygosity of the animals is very important. We designed a one day method based on the loss of a small portion of the mouse genome as a consequence of the transgene integration. We have sequenced this region and used selected fragments from 
it as templates for a PCR. Together with two control PCRs, one to detect the mouse Apo A1 region and one to detect the UPA transgene itself, this single tube PCR method meets the requirements mentioned above.

A search in the mouse genome database revealed that a region homologous $(p=8,9 \mathrm{E}-58)$ to the deleted sequence was located on chromosome 10, indicating that this is the integration site. Finally it must be stressed that with this method it is impossible to misclassify a negative or a heterozygous uPA mouse as a homozygous animal because the screening method is based on the absence of a signal. Any possible contamination of $\mathrm{uPA}^{+/+}$chromosomal DNA with, e.g., wild type DNA will result in a heterozygous result. This method thus prevents the occurrence of such errors and as a consequence thereof the waste of very scarce and valuable hepatocytes in erroneous transplantations.

\section{Acknowledgment}

The authors wish to thank Dr. R. Palmiter, Howard Hughes Medical Institute, University of Washington, for his kind donation of plasmid p3371C.

\section{References}

[1] G.M. Lauer, B.D. Walker, Hepatitis C virus infection, N. Engl. J. Med. 345 (2001) 41-52.
[2] J.A. Rhim, E.P. Sandgren, R.D. Palmiter, R.L. Brinster, Complete reconstitution of mouse liver with xenogeneic hepatocytes, Proc. Natl. Acad. Sci. USA 92 (1995) 49424946.

[3] J. Petersen, M. Dandri, S. Gupta, C.E. Rogler, Liver repopulation with xenogeneic hepatocytes in B and T cell-deficient mice leads to chronic hepadnavirus infection and clonal growth of hepatocellular carcinoma, Proc. Natl. Acad. Sci. USA 95 (1998) 310 315.

[4] D.F. Mercer, D.E. Schiller, J.F. Elliott, D.N. Douglas, C. Hao, A. Rinfret, W.R. Addison, K.P. Fischer, T.A. Churchill, J.R. Lakey, D.L. Tyrrell, N.M. Kneteman, Hepatitis $\mathrm{C}$ virus replication in mice with chimeric human livers, Nat. Med. 7 (2001) 927933.

[5] J.L. Heckel, E.P. Sandgren, J.L. Degen, R.D. Palmiter, R.L. Brinster, Neonatal bleeding in transgenic mice expressing urokinase-type plasminogen activator, Cell 62 (1990) 447456.

[6] J.A. Rhim, E.P. Sandgren, J.L. Degen, R.D. Palmiter, R.L. Brinster, Replacement of diseased mouse liver by hepatic cell transplantation, Science 263 (1994) 1149-1152.

[7] T.C. Weglarz, J.L. Degen, E.P. Sandgren, Hepatocyte transplantation into diseased mouse liver. Kinetics of parenchymal repopulation and identification of the proliferative capacity of tetraploid and octaploid hepatocytes, Am. J. Pathol. 157 (2000) 1963-1974.

[8] M. Dandri, M.R. Burda, E. Torok, J.M. Pollok, A. Iwanska, G. Sommer, X. Rogiers, C.E. Rogler, S. Gupta, H. Will, H. Greten, J. Petersen, Repopulation of mouse liver with human hepatocytes and in vivo infection with hepatitis B virus, Hepatology 33 (2001) 981-988.

[9] E.P. Sandgren, R.D. Palmiter, J.L. Heckel, C.C. Daugherty, R.L. Brinster, J.L. Degen, Complete hepatic regeneration after somatic deletion of an albumin-plasminogen activator transgene, Cell 66 (1991) 245-256.

[10] http://www.jax.org. 